\title{
Deformation of Face-Centered Cubic Polycrystal and Stress Tensor Components in Grains
}

\author{
Svetlana Kiseleva ${ }^{1, \mathrm{a})}$, Ivan Gibert ${ }^{2, \mathrm{~b})}$, Natalya Popova ${ }^{1, \mathrm{c})}$ and Nina Koneva ${ }^{1, \mathrm{~d})}$ \\ ${ }^{1}$ Tomsk State University of Architecture and Building, 2 Solyanaya Square, Tomsk 634003 Russian Federation \\ ${ }^{2}$ Tomsk Polytechnic University, 30 Lenina Avenue, Tomsk 634050 Russian Federation \\ a)corresponding author: kisielieva1946@mail.ru \\ b) gibert1993@mail.ru \\ c)natalya-popova-44@mail.ru \\ d)koneva@tsuab.ru
}

\begin{abstract}
The transmission electron microscopy (TEM) has been used to indicate the contribution of stress tensor components to internal stresses in deformed polycrystalline austenitic steel. TEM images of steel deformation demonstrate bend extinction contours the parameters of which allow detecting the stress tensor components (bending and torsional stresses of a crystal lattice). The diagrams are constructed for the contribution of stress tensor components to internal stresses in grains having different types of bending. The regularities are obtained for the stress distribution due to bending-torsion of a crystal lattice in the deformed face centered cubic crystal system. The experiment shows that the contribution of bending and torsion stress components to the internal stresses is different during the increase of deformation. The torsion stress component always grows with the increase of deformation, while the bending stress component either grows or lowers. The growth in the torsion stress component is typical for grains with compound bending.
\end{abstract}

\section{INTRODUCTION}

It is well known, that polycrystal deformation produces the internal stress fields inside its grains [1-5]. As a result, bend extinction contours are observed on the transmission electron microscopy (TEM) images of deformed material. The parameters of these extinction contours allow detecting the stress field characteristics [6]. The extinction contour is a point locus in which the atomic planes of the given family are parallel and, hence, are in the identical reflecting position. The extinction contours observed on TEM images reflect the nature of the local deformation of the specimen that can be both plastic and elasto-plastic [6, 7]. The behavior of the extinction contours reflects the bending-torsion amplitude of the crystal lattice observed in different local areas of material. The types of deformation of the crystal lattice, such as bending, torsion or mixed are identified by the mutual orientation of the extinction contour line and the reflection vector $\vec{g}$. The internal stress is the second-order tensor [8]. The stress tensor components are presented by the bending and torsion stress components of a crystal lattice. Little is currently known about their contributions to the internal stresses.

This paper mainly focuses on the investigations of the different grain bending affecting the stress tensor components in deformed austenitic steel. The stress tensor components are identified for the deformed polycrystal using the suggested methodology. Depending on the nature of deformation, either elastic and plastic or only plastic stress components are taken into account. Research results are discussed and compared with the experimental data obtained in the work by Kozlov et al. [9].

\section{IDENTIFICATION METHOD FOR STRESS TENSOR COMPONENTS IN DEFORMED MATERIAL}

The stress tensor component in deformed material is identified using the methodology described in the work by Kiseleva et al. [10] which has been multiply applied in works [11-13]. The parameters of extinction contours observed on TEM images allow detecting the bending-torsion components $\sigma_{11}$ and $\sigma_{12}$ of the crystal lattice. They equal respectively to 


$$
\begin{aligned}
& \sigma_{11}=\sigma_{11, p}+\sigma_{11, e l}, \\
& \sigma_{12}=\sigma_{12, p}+\sigma_{12, e l} . \\
& \sigma_{11, p}=\mu \sqrt{b \cdot \chi_{11, p}}, \\
& \sigma_{11, e l}=\mu \cdot t \cdot \chi_{11, e l}, \\
& \sigma_{12, p}=\mu \sqrt{b \cdot \chi_{12, p}}, \\
& \sigma_{12, e l}=\mu \cdot t \cdot \chi_{12, e l},
\end{aligned}
$$

where $\mu$ is the Young's modulus; $b$ is Burgers vector; $t$ is the foil thickness; $\sigma_{11}$ and $\sigma_{12}$ are stress tensor components; $\chi_{11, p}, \chi_{12, p}$ and $\chi_{11, e l}, \chi_{12, e l}$ are respectively bending and torsion tensor components:

$$
\begin{aligned}
& \chi_{11, p}=\frac{\Delta \varphi}{\Delta L_{x, p}}, \\
& \chi_{11, e l}=\frac{\Delta \varphi}{\Delta L_{x, e l}}, \\
& \chi_{12, p}=\frac{\Delta \varphi}{\Delta L_{y, p}}, \\
& \chi_{12, e l}=\frac{\Delta \varphi}{\Delta L_{y, e l}},
\end{aligned}
$$

where $\Delta \varphi$ is the angle of grain boundary misorientation on $\Delta L$ length; indices 11 and 12 are respectively for bending and torsion stress components.

\section{RESULTS AND DISCUSSION}

The type 1.1C-13Mn-Fe austenitic steel was investigated in this experiment. The test machine was used to measure tensile strength of steel specimens at $3.4 \cdot 10^{-4} \mathrm{~s}^{-1}$ speed. The specimens $5 \times 15 \times 75 \mathrm{~mm}$ in size were tested at room temperature up to the material rupture $(\varepsilon=36 \%)$. The average grain size was $34 \mu \mathrm{m}$. The steel specimens were quenched at $1050^{\circ} \mathrm{C}$ during $30 \mathrm{~min}$ and then subjected to deformation. The degree of deformation was detected by the length of the investigated specimen area. The electrospark discharge machine was used to cut off metal plates $0.25-0.35 \mathrm{~mm}$ thick perpendicular to the specimen axis of tension. The spark cutting mode was selected so that not to cause the additional distortions in the material structure.

The specimen surface was prepared in three stages. First, it was cleaned after cutting by hydrogen peroxide $\left(\mathrm{H}_{2} \mathrm{O}_{2}\right)$ mixed with a few drops of hydrofluoric acid (HF). Second, at room temperature the specimens were subjected to electrolytic polishing in the oversaturated solution of chromic anhydride containing orthophosphoric acid to obtain $\sim 0.2 \mu \mathrm{m}$ thick foil. Finally, the specimens were polished in the electrolyte foam at 60 $80^{\circ} \mathrm{C}$. Observations of the structure and phase composition on thin steel foils were made on the EM-125 transmission electron microscope and the attached goniometer. Specifications for the EM-125 included $125 \mathrm{kV}$ accelerating voltage and $\times 4000-\times 80000$ magnification. TEM images of the fine structure of material were used to detect the internal stresses.

TEM investigations are carried out for polycrystal grains under the different inclinations of the goniometer. TEM images presented in Fig. 1, 2 and 3 illustrate the structure of austenitic steel with dislocations. Bend extinction contours observed on these images indicate the presence of local stresses inside the grains. Two deformation degrees of $\varepsilon=14 \%$ and $\varepsilon=25 \%$ are shown in Fig. $1 a-d, 2 a, b$ and $3 a, b$. The vector of actual reflection $\vec{g}$ is normal to the goniometer axis. The investigated foil area includes neither interfaces nor misorientations within the displacement of extinction contours. The displacement is different at different foil inclinations. Thus, in Fig. $1 a, b$ and $2 a, b$, this displacement is parallel, while in Fig. $1 c, d$ and 3 a, $b$ the contours either approach or move apart. In the first case, the grain bending is simple, in the second it is compound. Compound bending can cover two or more planes of the crystal lattice. 
Figures 4 and 5 contain plots of the contributions of bending and torsion stress components to internal stresses in grains with different bending. The analysis of these contributions shows that these block diagrams are

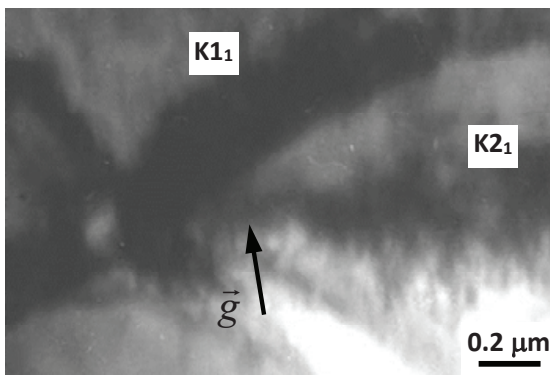

a)

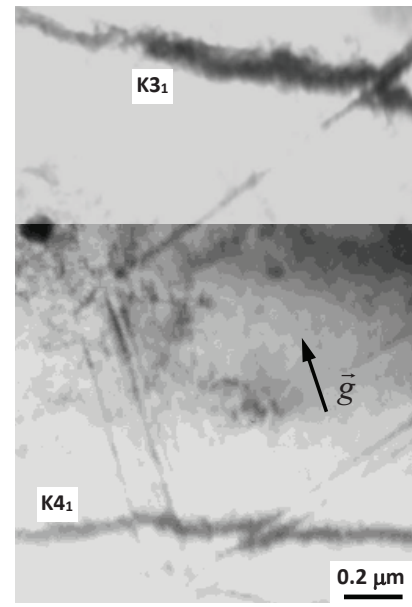

c)

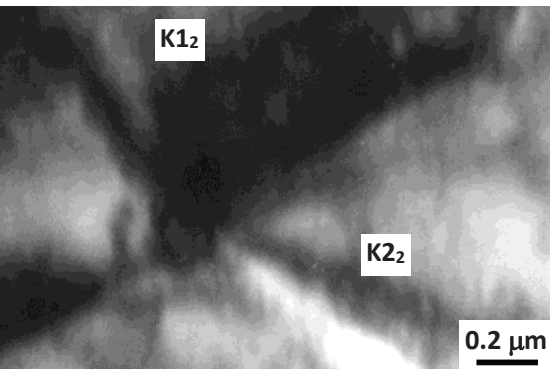

b)

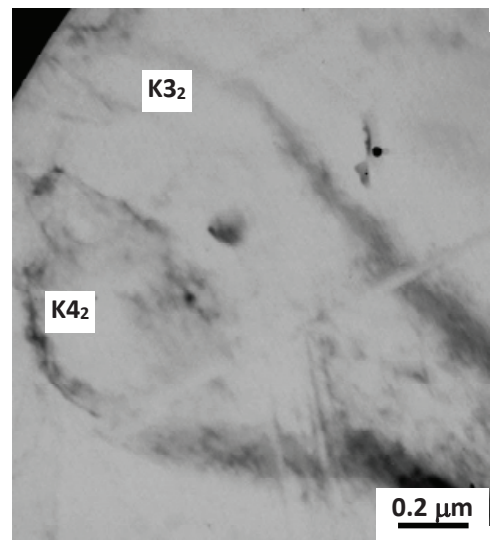

d)

FIGURE 1. TEM images of tensile deformation $(\varepsilon=14 \%)$ of steel specimen at different inclinations of the goniometer axis: a) $1^{0}$; b) $3^{0}$; c) $0^{0}$; d) $10,5^{0} ; K 1_{1}, K 2_{1} ; K 1_{2}, K 2_{2} ; K 3_{1}, K 4_{1} ; K 3_{2}, K 4_{2}$ - bend extinction contours; $\vec{g}$ - diffraction vector

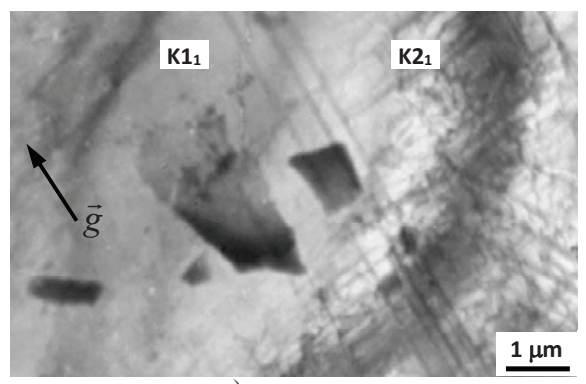

a)

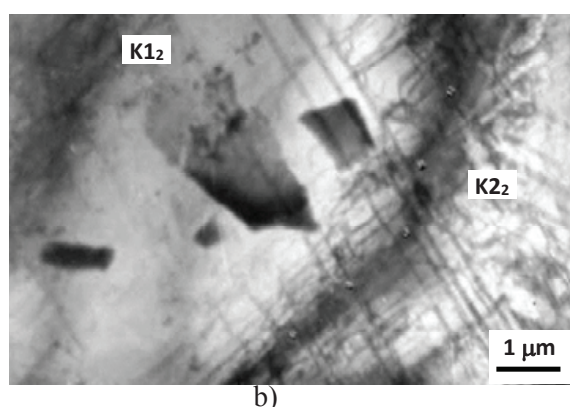

b)

FIGURE 2. TEM images of tensile deformation $(\varepsilon=25 \%)$ of steel specimen at different inclinations of the goniometer axis: a) $8.8^{0}$, b) $9.2^{0} ; \mathrm{K} 1_{1}, K 2_{1} ; K 1_{2}, K 2_{2}$ - bend extinction contours; $\vec{g}$ - diffraction vector

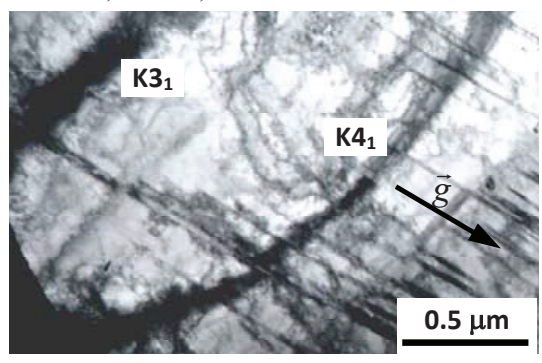

a)

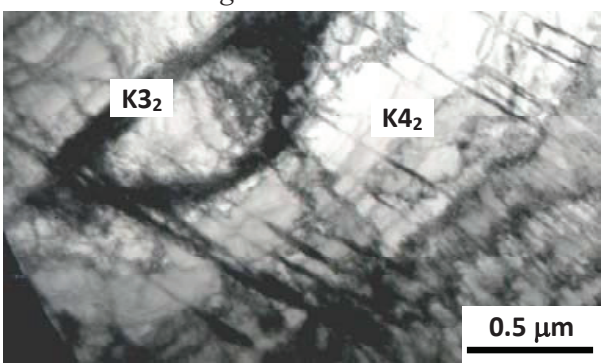

b)

FIGURE 3. TEM images of tensile deformation $(\varepsilon=25 \%)$ of steel specimen at different inclinations of the goniometer axis: a) $0^{0}$, b) $3^{0} ; K 3_{1}, K 4_{1}, K 3_{2}, K 4_{2}$ - bend extinction contours; $\vec{g}$ - diffraction vector 


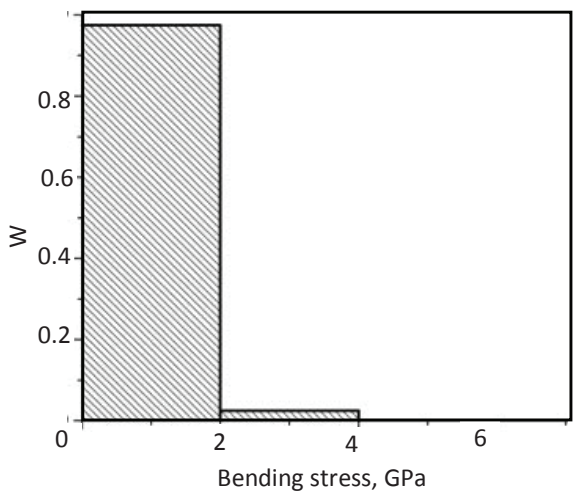

a)

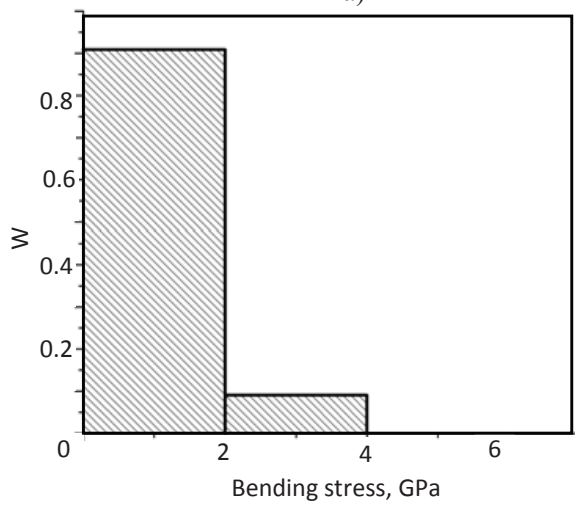

c)

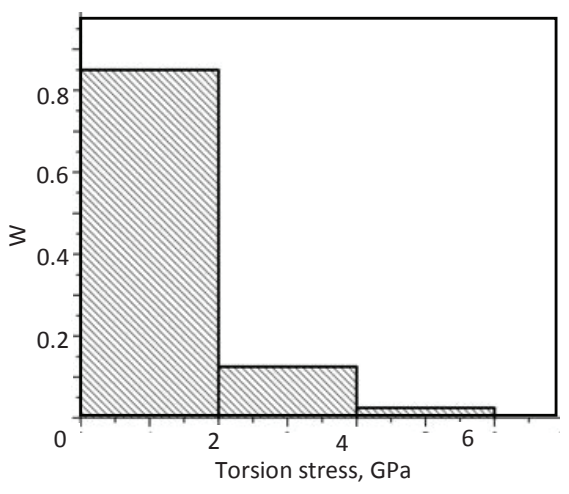

b)

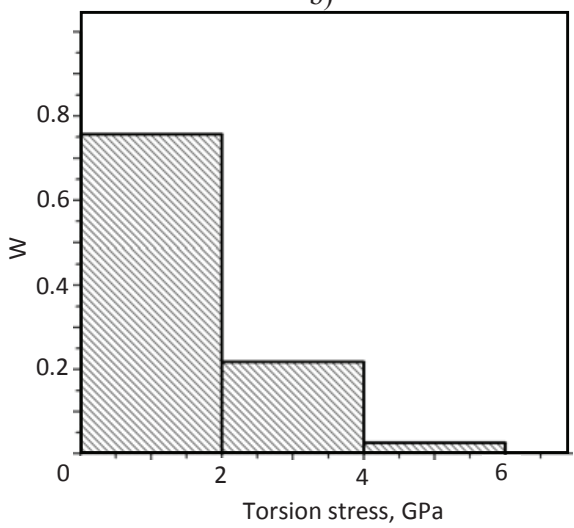

d)

FIGURE 4. Block diagrams of contribution $\mathrm{W}$ of internal stress tensor components $(\varepsilon=14 \%)$ for grains: a, b ) with simple bending; c, d) with compound bending.

multimodal for both simple and compound grain bending. Multimodality of the block diagrams indicates to the presence of elasto-plastic deformation in the specimen local areas. In all cases, the number of local areas with the bending-torsion stress components $\sigma_{11}$ and $\sigma_{12}$ not exceeding $2 \mathrm{GPa}$, is larger than that exceeding $2 \mathrm{GPa}$. For the torsion stress component, the number of local areas of $\sigma_{12}>2 \mathrm{GPa}$ grows with the increase of deformation $(\varepsilon=14 \%$ and $25 \%$ ). This is more distinctly observed in grains with compound bending. The detected values of bending and torsion stress components are given in Tables 1 and 2.

TABLE 1. Stress tensor component contributions to internal stresses in grains with different bending, $\varepsilon=14 \%$

\begin{tabular}{lccc}
\hline \multicolumn{1}{c}{ Bending type } & Bending stress $\boldsymbol{\sigma}_{\mathbf{1 1}}, \mathbf{G P a}$ & Torsion stress $\boldsymbol{\sigma}_{\mathbf{1 2}}, \mathbf{G P a}$ & Inside grain $<\boldsymbol{\sigma}>, \mathbf{G P a}$ \\
\hline Simple & 0.46 & 1.00 & 1.11 \\
Compound & 0.85 & 1.43 & 1.67 \\
\hline
\end{tabular}

TABLE 2. Stress tensor component contributions to internal stresses in grains with different bending, $\varepsilon=25 \%$

\begin{tabular}{lccc}
\hline \multicolumn{1}{c}{ Bending type } & Bending stress $\boldsymbol{\sigma}_{\mathbf{1 1}}, \mathbf{G P a}$ & Torsion stress $\boldsymbol{\sigma}_{\mathbf{1 2}}, \mathbf{G P a}$ & Inside grain $<\boldsymbol{\sigma}>\mathbf{, ~ G P a}$ \\
\hline Simple & 0.93 & 1.16 & 1.50 \\
Compound & 0.84 & 1.32 & 1.57 \\
\hline
\end{tabular}

The torsion stress $\sigma_{12}$ in grains with compound bending is higher than in grains with simple bending that can be seen in Tables 1 and 2 for respectively $\varepsilon=14 \%$ and $\varepsilon=25 \%$ deformation. At the same time, the bending stress $\sigma_{11}$ behaves differently, i.e. at $\varepsilon=14 \%$, it is higher in grains with compound bending, while at $\varepsilon=25 \%$ it is lower in grains with simple bending. Thus, the torsion stress $\sigma_{12}$ is always higher in grains with compound bending. Therefore, it is believed that the increase in the torsion stress $\sigma_{12}$ defines the compound bending of grains with the growth of the deformation degree. 


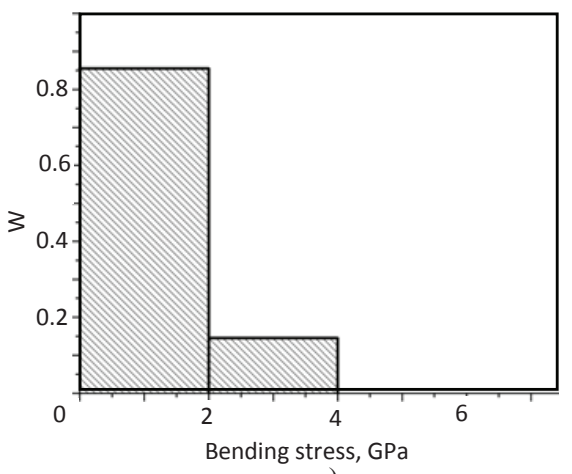

a)

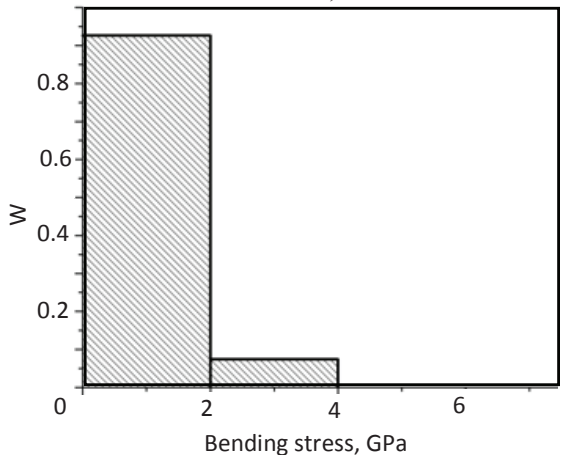

c)

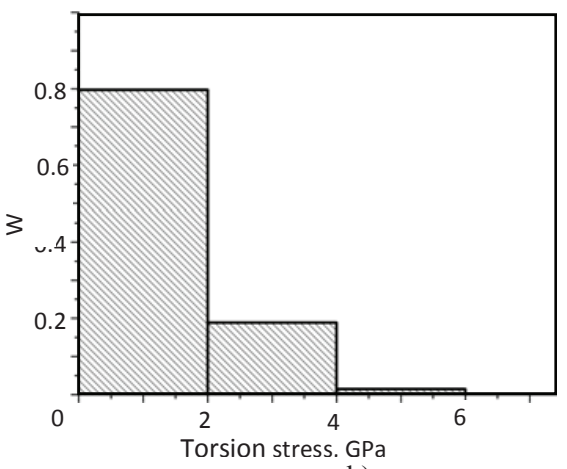

b)

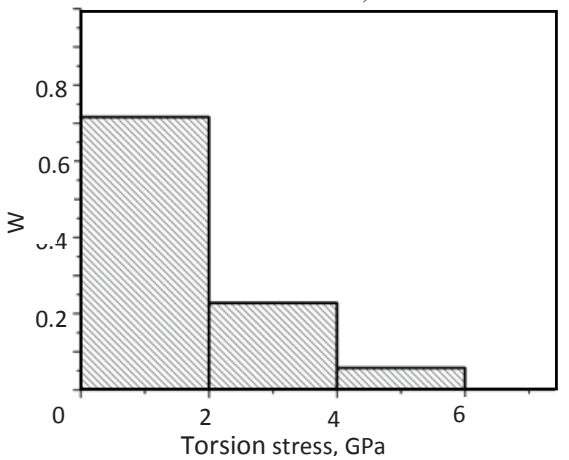

d)

FIGURE 5. Block diagrams of contribution $W$ of internal stress tensor components $(\varepsilon=25 \%)$ for grains: $a, b)$ with simple bending; c, d) with compound bending.

\section{CONCLUSION}

During the deformation of polycrystalline austenitic steel the formation of grains with simple and compound bending was observed. The experiment showed that the contribution of bending and torsion stress components to the internal stresses is different during the increase of deformation. With the growth of the deformation degree, the torsion stress component always increased, while the bending stress component either increased or decreased. It was shown that at one and the same degree of deformation, the torsion stress component was always higher in grains with compound bending than in grains with simple bending. Thus, the higher torsion stress component is specific to the grains with compound bending.

\section{ACKNOWLEDGMENTS}

The work was performed within the framework of governmental contract N 3.295.2014/K of Education and Science of the Russian Federation and Grant N 16-48-700198 from the Russian Foundation for Basic Research.

\section{REFERENCES}

1. N. A. Koneva and Ed. V. Kozlov, "Internal stress fields in ultrafinegrained materials", in Structural-Phase Conditions and Properties of Metallic Systems, edited by A. I. Potekaev (NTL Publisher, Tomsk, 2004) (in Russian), pp.83-110.

2. V. E. Panin, A. V.Panin, T. F. Elsukova and Yu. F. Popkova. Phys. Mesomech. (in Russian) 17, 6, 7-18 (2014).

3. V. E. Shavshukov, Phys. Mesomech. (in Russian) 15, 6, 85-91 (2012).

4. I. A. Ditenberg, A. N. Tyumentsev, A. V. Korzninikov and E. A. Korzninikova, Phys. Mesomech. (in Russian) 15, 5, 59-68 (2012).

5. A. N. Tyumentsev, I. A. Ditenberg, A. D. Korotaev and K. I. Denisov, Phys. Mesomech. (in Russian) 16, 3, 63-9 (2013). 
6. E. V. Kozlov, D. V. Lychagin, N. A. Popova and et al., "Far-ranging stress fields and their effect on deformation of structurally non-uniform materials", in Physics of strength of heterogeneous materials, edited by A. E. Romanov (Ioffe Inst. Publ., Leningrad, 1988) (in Russian), pp. 3-13.

7. N. A. Koneva, E. V. Kozlov, L. I. Trishkina and D. V. Lychagin "Long-range stress fields, bending-torsion amplitude of the crystal lattice and stages of plastic deformation", in New Mechanical and Physical Research Methods of Materials under Loading, edited by V. E. Panin (TSU Publ., Tomsk, 1990), pp. 8392.

8. A. A. Smirnov, Molecular-kinetic Theory of Metals (Nauka Publ, Moscow, 1966) (in Russian), 276 p.

9. E. V. Kozlov, N. A. Koneva, N. A. Popova, "Hadfield steel hardening mechanisms", in Modern Tendencies of Structural and Property Modification of Materials, edited by N. N. Koval' and V. E. Gromov (NTL Publ., Tomsk, 2015) (in Russian), pp. 312-330.

10. S. F. Kiseleva, N. A. Popova, N. A. Koneva and Ed. V. Kozlov, Fundamental Problems of Modern Mater. Sci. (in Russian) 9, 1, 7-14 (2012).

11. S. F. Kiseleva, N. A. Popova, N. A. Koneva and Ed. V. Kozlov, Adv. Mater. Res. Trans. Tech. Publication, Switzerland. 1013, 72-76 (2014).

12. I. Gibert, S. Kiseleva, N. Popova, N. Koneva and E. Kozlov, Adv. Mater. Res. 1013, 133-137 (2014).

13. I. Gibert, S. Kiseleva, N. Popova, N. Koneva, and E. Kozlov. AIP Conf. Proc. 1698, 020004 (2016). 